\title{
Adhesion-Free Thin-Film-Like Curvature Sensors Integrated on Flexible and Wearable Electronics for Monitoring Bending of Joints and Various Body Gestures
}

\author{
Hao Liu, Hongyu Zhao, Shuang Li, Jianqiao Hu, Xinran Zheng, Rui Li, * Yuli Chen, * \\ and Yewang Su*
}

Flexible and wearable electronics integrated with various sensors have great potential for applications in monitoring human activities and personal health. Bending and tension/compression dominate the deformation modes yielded by flexure of joints and diverse body gestures. A key challenge now is to sense the curvature/bending angle, while much research has been focused on the strain sensors for tension/compression. Alternative approaches by strain sensors or noncontact optical methods for curvature sensing are not practical for wearable electronics. A novel adhesionfree thin-film-like curvature sensor that can monitor bending activities is introduced for flexible and wearable electronics. This study presents comprehensive design, fabrication, mechanism, structural analysis, performance characterization, and device-level demonstrations for bending of joints, gesture recognition, and real-time sitting posture correction. The most prominent advantage of the present sensor is that the measurement is independent of the strain of the target surface and the interfacial slippage, thus the perfect adhesion between the sensor and the surface is unnecessary. The features of adhesion-free, simple mechanical principle, low cost, and satisfactory monitoring results highlight the superiority of the present curvature sensors for practical applications to flexible and wearable electronics.

\section{Introduction}

Various pressure, ${ }^{[1]}$ thermal, ${ }^{[2]}$ chemical, ${ }^{[3]}$ and strain sensors ${ }^{[4-7]}$ integrated with flexible and stretchable platforms represent an important branch of the nextgeneration electronics, with great potential for applications in monitoring human activities and personal health. The dominated deformation modes describing diverse body gestures and activities can be normally categorized into two groups: tension/compression and bending. Accordingly, wearable strain sensors and curvature sensors must be with large sensing range because of the large deformation yielded by varous activities. Till now, there have been many investigations focusing on the strain sensors that can monitor large tension/compression deformation, and the primary strategies are attempting various materials as sensing elements, including silicon nanoribbons, ${ }^{[8]}$ metal nanowires,,$^{[9]}$ carbon blacks, ${ }^{[10,11]}$ carbon nanotubes, ${ }^{[4,12,13]}$ graphene, ${ }^{[14,15]}$
H. Liu, H. Zhao, S. Li, Prof. J. Hu, Prof. Y. Su

State Key Laboratory of Nonlinear Mechanics

Institute of Mechanics

Chinese Academy of Sciences

Beijing 100190, China

E-mail: yewangsu@imech.ac.cn

H. Liu, Prof. Y. Chen

Institute of Solid Mechanics

Beihang University (BUAA)

Beijing 100191, China

E-mail: yulichen@buaa.edu.cn

H. Zhao, S. Li, Prof. Y. Su

School of Engineering Science

University of Chinese Academy of Sciences

Beijing 100049, China

The ORCID identification number(s) for the author(s) of this article can be found under https://doi.org/10.1002/admt.201800327.
X. Zheng, Prof. R. Li

State Key Laboratory of Structural Analysis for Industrial Equipment

Department of Engineering Mechanics

and International Research Center for Computational Mechanics

Dalian University of Technology

Dalian 116024, China

E-mail: ruili@dlut.edu.cn

Prof. Y. Chen

Department of Civil and Environmental Engineering

Northwestern University

Evanston, IL 60208, USA

DOI: 10.1002/admt.201800327 

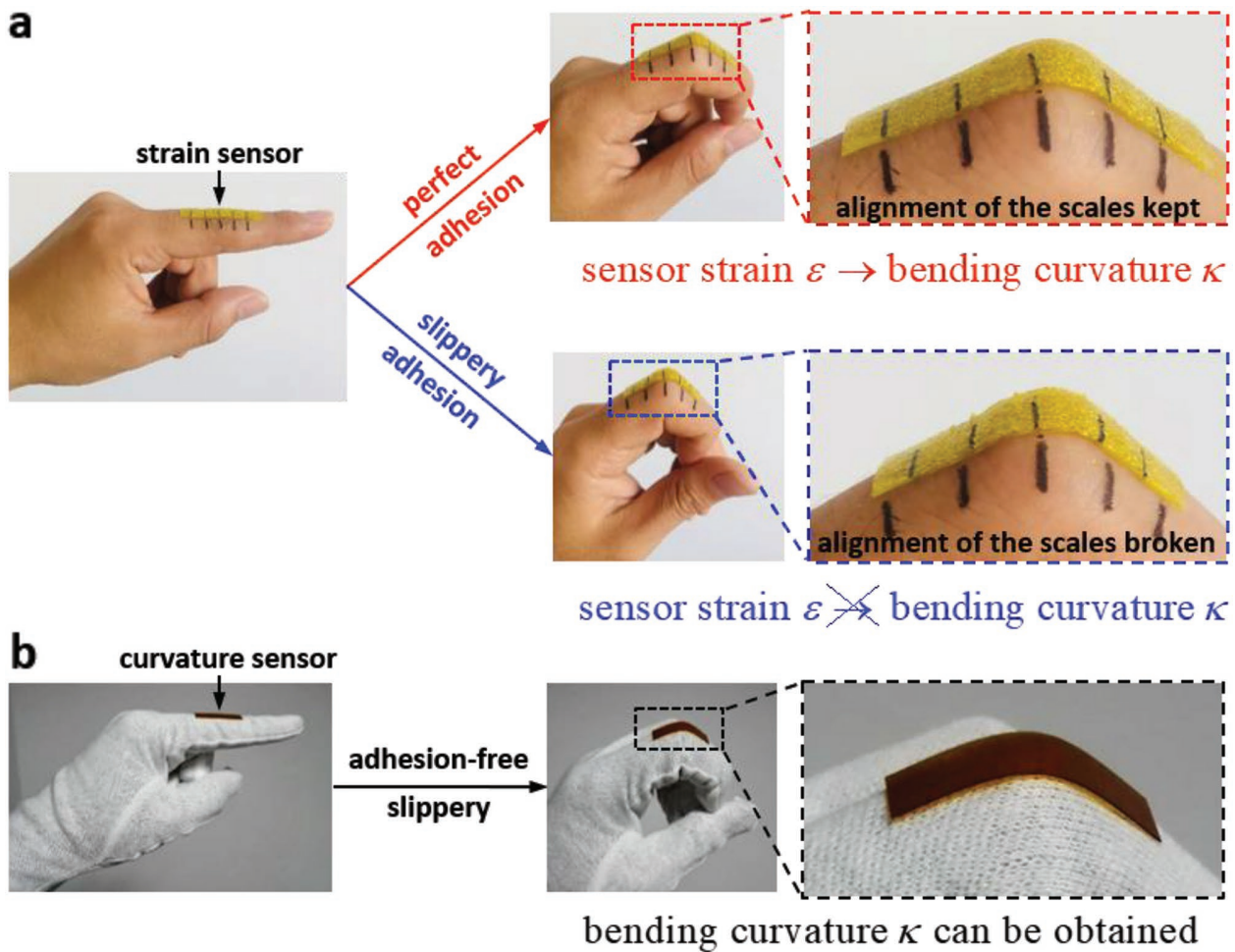

Figure 1. a) Schematic illustration of both perfect and slippery adhesions of a strain sensor to a joint of an index finger for measurement of bending deformation. b) Curvature sensor that is attached to a glove to achieve adhesion-free curvature measurement.

carbonized silk fabrics, ${ }^{[16]}$ etc. Basically, wearable sensors that include ultrathin thickness and low system-level modulus are good candidate for monitoring of human activities with high signal to noise ratio. ${ }^{[17]}$ Also, fully rubber conductive materials that show quite good van der waals adhesion to the human skin have been used for wearable sensing/actuating applications. ${ }^{[18]}$ However, in comparison with the prosperity of research on strain sensors, there have been rare reports on the curvature sensors that can measure bending deformation, although accurate dynamic measurement of curvature is of great interest: it is especially useful for detection of activities of some special body parts such as spines and finger/elbow/knee/ankle joints.

For characterising bending deformation, using strain sensors may serve as an alternative approach, ${ }^{[19-21]}$ but the measurement result is strongly sensitive to the types of adhesion between the sensors and the target surface (e.g., the human skin). Figure 1a gives a schematic illustration of both perfect and slippery adhesions of a strain sensor to a joint of an index finger. The two types of adhesion are differentiated according to whether there is slippage on the finger during bending, which is characterized by whether alignment of the scales on the finger and strain sensor before bending of the finger is kept after its bending. For perfect adhesion, the bending curvature/angle can be obtained from the sensor strain $\varepsilon$ because they have one-to-one correspondence, whereas it cannot be captured in slippery adhesion because it is difficult to quantify the effect of the slippage. This can be shown by an extreme case in which $\varepsilon$ is zero (nonstretchable) but the bending curvature $\kappa$ is nonzero: the nonzero bending curvature/angle cannot be deduced by the measured zero sensor strain. Therefore, the alternative approach by strain sensors demands perfect adhesion of the sensors to target surfaces, which is a harsh requirement and severely limits its practical applications to wearable electronics. Figure $1 \mathrm{~b}$ shows our curvature sensor that is attached to a glove to achieve adhesion-free curvature measurement: even though there is slippage between the finger and glove, the bending curvature can still be obtained; this will be elaborated in Section 2.3. On the other hand, the most existing techniques developed for curvature sensing are focused on noncontact optical methods ${ }^{[22]}$ and fiber-optic sensors. ${ }^{[23,24]}$ However, complex measurement systems and complicated operation principles have hindered their portability and applications to wearable devices. Therefore, it is indispensable to develop new curvature sensors that are suitable for integration with flexible and wearable electronics.

Here, we introduce a novel adhesion-free thin-film-like curvature sensor that can effectively monitor bending activities. The key idea of the design is based on the mechanism of beam bending, of which, the curvature can be deduced from the strain difference of the top and bottom surfaces, and is independent of the strain values themselves. From the view of applications, the most prominent advantage of the present sensor is that the measurement is independent of the strain of the target surface and the interfacial slippage, thus the perfect adhesion between the sensor and the surface is unnecessary; this is very attractive for wearable bioelectronics. In the following, we present design, fabrication, mechanism, structural analysis, performance characterization, and device-level demonstrations in representative applications of adhesion-free curvature sensors. Satisfactory experimental results, the features of adhesion-free, low-cost, simple operation principle, and portability guarantee 
the tremendous application potential in flexible and wearable electronic devices.

\section{Results and Discussion}

\subsection{Design and Fabrication of Novel Curvature Sensor}

Figure 2a shows an exploded view of the developed curvature sensor; the multilayer configuration is composed of an interlayer, two sensitive grids with the external leads located at the top and bottom of the interlayer, and two encapsulated layers at the top and bottom of the structure, respectively. The materials used include the constantan foil for the sensitive grids, phenolic resin for the interlayer, polyimide (PI) for the encapsulated layers, and slender copper wire for the external leads. Constantan has the best overall combination of properties, including, e.g., an adequately high strain sensitivity, which is relatively insensitive to strain level and temperature. Its resistivity is high enough to achieve suitable resistance values in even very small grids, and its temperature coefficient of resistance is not excessive. Besides, constantan is characterized by
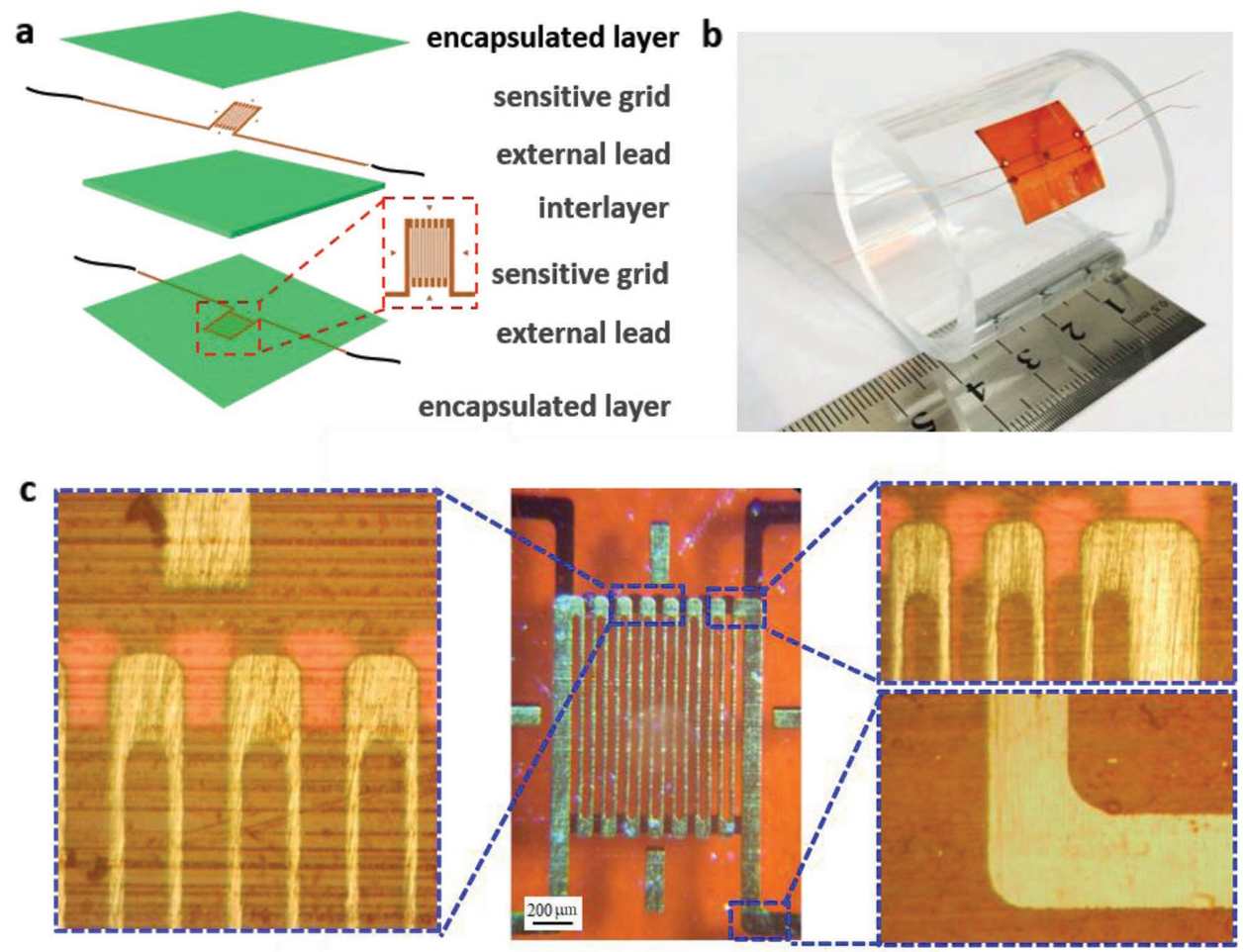

d
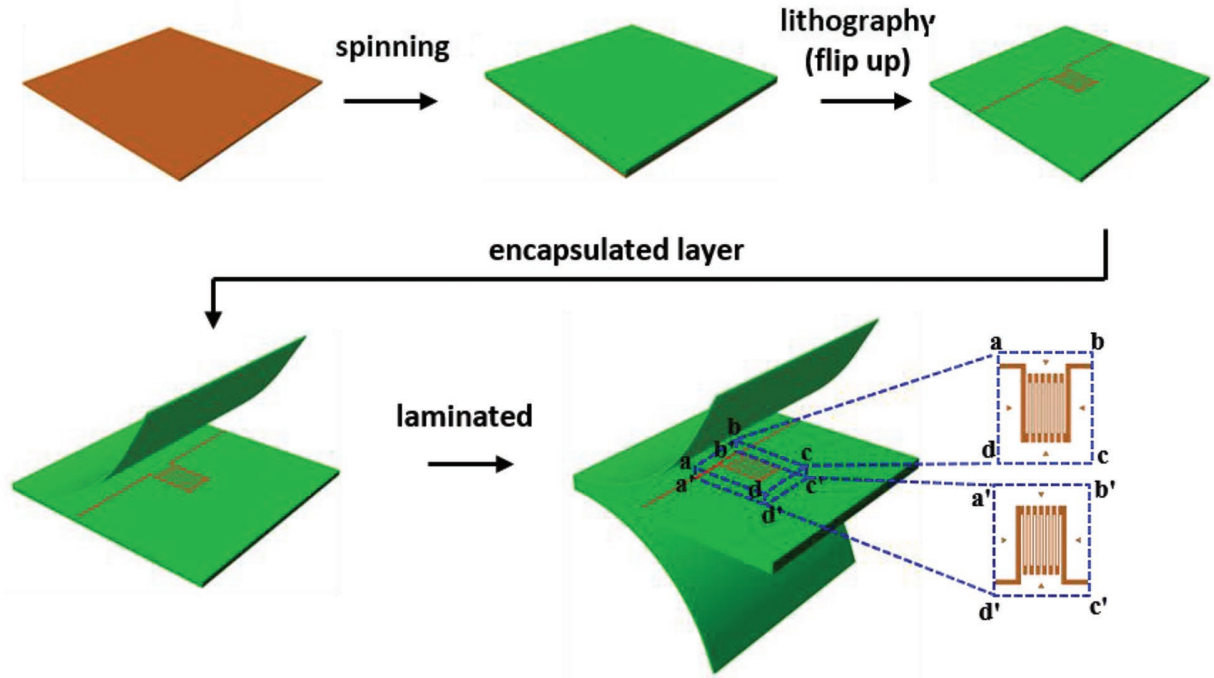

Figure 2. Schematic illustration of the novel flexible curvature sensor. a) Exploded view of the sensor. b) Demonstration of a curvature sensor that is attached to a cylinder. c) Top-view optical image of the sensitive grids. d) Fabrication process of a curvature sensor (described in detail in the main text). 
good fatigue life and relatively high elongation capability, which is favorable when applied to flexible electronics. Figure $2 \mathrm{~b}$ demonstrates a fabricated $1.5 \mathrm{~cm} \times 1.5 \mathrm{~cm}$ curvature sensor that is attached to a cylinder. The sensitive grids have the apparent inplane dimensions of $1.2 \mathrm{~mm} \times 1 \mathrm{~mm}$, with details shown in Figure 2c. More detailed configuration and dimensions appear in Figure S1 and Table S1 (Supporting Information), respectively. The fabrication process of a curvature sensor is outlined in Figure $2 d$, which is realized by conventional processing strategies. The main steps include spin coating, lithography, encapsulation, and lamination. Details appear in the Experimental Section. Since the manufacturing procedure could be achieved by usual equipment, it has great potential for realizing low-cost curvature sensors.

\subsection{Mechanism, Structural Analysis, and Characterization of the Curvature Sensor}

The structure of the developed curvature sensor is designed according to the mechanism of beam bending. ${ }^{[25]}$ It is known that the convex surface of a bent beam is in tension while the concave surface is in compression. If the lengthwise strains of both the surfaces are obtained, the bending curvature of the beam can be inferred by the beam theory. Since the strain can be related to the measurable resistance change that is extracted by a sensitive grid, the measurement of bending curvature becomes possible. Figure 3a shows the schematic diagram of our curvature sensor under pure bending. In the present layout, under the beam theory-based deformation mode with plane cross-section, the bending curvature, denoted by $\kappa$, is expressed by ${ }^{[25]}$

$\kappa=\frac{\varepsilon_{\text {top }}-\varepsilon_{\text {bottom }}}{t}$

where $t$ is the thickness of the interlayer that is much thicker than the sensitive grids, $\varepsilon_{\text {top }}$ and $\varepsilon_{\text {bottom }}$ are the strains of the top and bottom sensitive grids, respectively. For the sensitive grids, we have the relationship, $\Delta R / R=K_{\mathrm{S}} \varepsilon$, where $\Delta R$ is the change in resistance caused by the strain $\varepsilon, R$ the resistance of the undeformed grid, and $K_{\mathrm{S}}$ the gauge factor $(\mathrm{GF}) \cdot{ }^{[4,12,14,16,26]}$ Accordingly,

$\frac{\Delta R_{\text {top }}}{R}=K_{\mathrm{S}} \varepsilon_{\text {top }}$
$\frac{\Delta R_{\text {bottom }}}{R}=K_{\mathrm{s}} \varepsilon_{\text {bottom }}$

are obtained for the top and bottom sensitive grids, respectively, where $\Delta R_{\text {top }}$ and $\Delta R_{\text {bottom }}$ are the changes in resistance. From Equations (1) and (2), the curvature can be given by the directly tested parameters

$\kappa=\frac{1}{t K_{\mathrm{S}} R}\left(\Delta R_{\mathrm{top}}-\Delta R_{\mathrm{bottom}}\right)$

If the sensor is subject not only to bending, but also to stretching in use, the strain in each of the top and bottom layers contains both bending strain and membrane strain, i.e., a
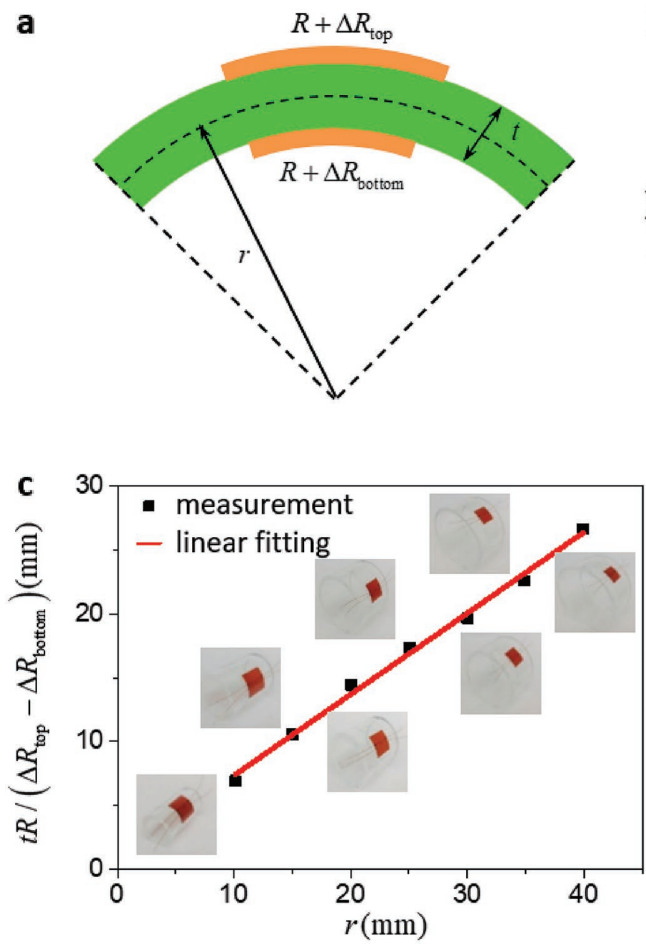
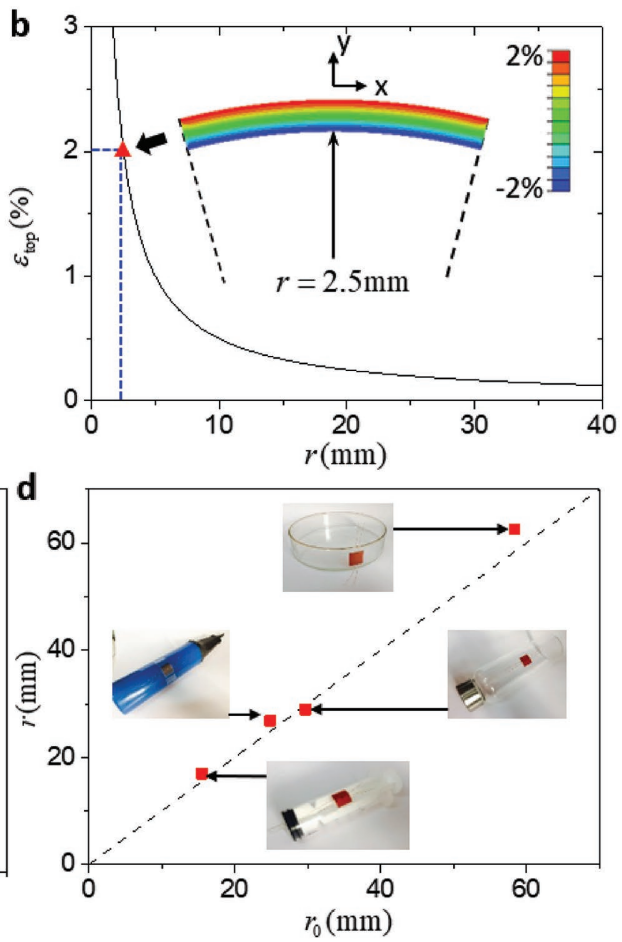

Figure 3. a) Schematic illustration of a curvature sensor under pure bending. b) Relationship between the strain in the top sensitive grid and radius of curvature. c) Difference of resistance changes in the two sensitive grids versus the cylinder radius. d) Comparison of the measured radii of curvature and those real of typical objects, including an inflator, an injector, a bottle, and a petri dish. 
$\varepsilon_{\text {top }}=\varepsilon_{\text {membrane }}+\varepsilon_{\text {bending }}$, and $\varepsilon_{\text {bottom }}=\varepsilon_{\text {membrane }}-\varepsilon_{\text {bending, }}$, where the bending strains in the top layer ( $\varepsilon_{\text {bending }}$ ) and bottom layer $\left(-\varepsilon_{\text {bending }}\right)$ are opposite. The effect of membrane strain induced by stretching is eliminated by the difference between $\varepsilon_{\text {top }}$ and $\varepsilon_{\text {bottom }}$, as revealed in Equations (1)-(3). As a result, whether the sensor is stretched or not does not impact the curvature measurement. As illustrated in introduction (see Figure 1), the usual strategy that using strain sensors for curvature measurements needs perfect adhesion of the sensors to target surfaces or enough interfacial friction (i.e., the sensor must be stretched with stretching of the target surfaces), which is impractical for wearable electronics because the slippage (or even nonadhesion) is usually inevitable. Here, perfect adhesion is not required, as the curvature measurement is independent of the membrane strain. The present curvature sensor provides a very attractive feature, with great potential in achieving flexible and stretchable wearable electronic devices.

We adopt a part of the sensor, with $1.2 \mathrm{~mm}$ apparent length (the same size as that of the sensitive grids), $100 \mu \mathrm{m}$ thick interlayer, $10 \mu \mathrm{m}$ thick encapsulations, and $4 \mu \mathrm{m}$ thick sensitive grids (see Figure S1 and Table S1, Supporting Information for details), for the theoretical and finite element analysis (FEA, see the subsection "Finite Element Analysis" in the Experimental Section for details). According to the beam theory, ${ }^{[20]}$ the strains in the top and bottom sensitive grids (Figure 3a), depending on the radius of curvature $r(=1 / \kappa)$, are given by

$$
\left.\begin{array}{c}
\varepsilon_{\text {top }} \\
\varepsilon_{\text {bottom }}
\end{array}\right\}= \pm \frac{t}{2 r}
$$

The relationship between $\varepsilon_{\text {top }}$ and $r$ is plotted in Figure 3b, indicating that the bending radius should not be less than $2.5 \mathrm{~mm}$ to avoid overstrain (the present sensor offers $\approx 2 \%$ limit strain). According to Equation (3), the GF for the curvature sensor is defined as $K_{\mathrm{S}}=\left(\Delta R_{\text {top }}-\Delta R_{\text {bottom }}\right) /(t \kappa R)$. Calibration of $K_{\mathrm{S}}$ is implemented by measuring a set of cylinders with different known radii. A good linear correlation is found in Figure $3 c$ by plotting the difference of resistance changes in the two sensitive grids versus the cylinder curvature. Wheatstone bridge is incorporated in the read-out technique for a high accuracy measurement. Linear least-squares regression of all measurement points is used to determine $K_{\mathrm{S}}$ as 1.578 (reciprocal of the slope of the line). Using the calibrated curvature sensor, some objects, with prescribed radii of curvature ranging from $\approx 15$ to $60 \mathrm{~mm}$, are examined, including an inflator, an injector, a bottle, and a petri dish. Figure $3 \mathrm{~d}$ shows the measured radii $(r)$ versus those real $\left(r_{0}\right)$ of the objects by the dots with the coordinates $\left(r_{0}, r\right)$, where the dashed line corresponds to $r=r_{0}$. It is seen that almost all the dots are on the line, suggesting that the measured radii of curvature are accurate enough.

It should be emphasized that the present sensor is, of course, applicable to simultaneous measurement of curvature and tension/compression, with the latter simply accomplished according to $\varepsilon_{\text {bottom }}=\Delta R_{\text {bottom }} /\left(K_{\mathrm{S}} R\right)$.

\subsection{Adhesion-Free Tests and Sample Curvature Measurements}

Flexible and stretchable wearable electronic devices are either directly adhered to the human body or attached to those people wear such as the gloves and clothes. ${ }^{[4,27-29]}$ The latter cases have more extensive potential applications, since they are more acceptable for the users. However, the latter cases usually require the adhesion-free feature that the sensors can be integrated onto the gloves and clothes, but the measurement does not depend on the slippage between the wears and human body. Fortunately, as has been mentioned above, the present curvature sensor can achieve adhesion-free measurements due to its main advantage, i.e., independence of the strain of the target surface and dependence on bending deformation only. To validate this, we carry out some tests by attaching the sensor to a steel sheet for measuring bending deformation (Figure $4 a-h$ ).

Figure $4 \mathrm{a}, \mathrm{b}$ shows the flat and bent states of a sheet induced by compression in the length direction. According to the theory of Euler buckling, the radius of curvature at the center of the buckled steel sheet is ${ }^{[25,30]}$

$r=\frac{L}{2 \pi} \sqrt{\frac{L}{\Delta L}}$

where $L$ is the length of the undeformed sheet and $\Delta L$ the axial displacement of the end. The beam theory shows that the surface strain $\varepsilon_{\text {surface }}$ is proportional to the thickness of the steel sheet $t_{\text {sheet }}$, i.e., $\varepsilon_{\text {surface }}=t_{\text {sheet }} /(2 r)$, which, together with Equation (5), gives

$\varepsilon_{\text {surface }}=\frac{\pi t_{\text {sheet }}}{L} \sqrt{\frac{\Delta L}{L}}$

The curvature sensor is attached at the center of the steel sheet for measment. Two ways of attaching are investigated, one with the sheet bent to have a nonzero curvature before the sensor is attached (case I), the other one with the sensor attached before the sheet is bent (case II). These two cases lead to different strains in the sensors. For Case I, only bending strain exists, i.e., $\varepsilon_{\text {top }}=t /(2 r)$ and $\varepsilon_{\text {bottom }}=-t /(2 r)$ according to Equation (4). For case II, besides bending deformation, the surface strain $\varepsilon_{\text {surface }}$ of the steel sheet is also transfered to the sensor as a stretch. Both bending and membrane strains exist, i.e., $\varepsilon_{\text {top }}=t /(2 r)+\varepsilon_{\text {surface }}$ and $\varepsilon_{\text {bottom }}=-t /(2 r)+\varepsilon_{\text {surface }}$. According to the relationship from Equation (2), the measured resistance change for the two cases should also be different. The above derivations give

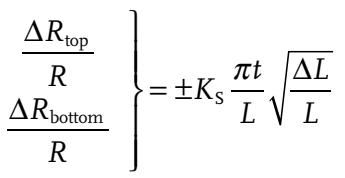

and

$$
\frac{\Delta R_{\text {bottom }}}{R}= \pm K_{\mathrm{S}} \frac{\pi t}{L} \sqrt{\frac{\Delta L}{L}} \underbrace{+K_{\mathrm{S}} \frac{\pi t_{\text {sheet }}}{L} \sqrt{\frac{\Delta L}{L}}}_{\text {effects of } \varepsilon_{\text {surface }}}
$$

Figure $4 \mathrm{c}$ shows the experimentally recorded $\Delta R_{\mathrm{top}} / R$ (upper red and green hyphens) and $\Delta R_{\text {bottom }} / R$ (lower red and green hyphens) for $\Delta L / L=0.2,0.4$, and 0.6 , respectively. The 

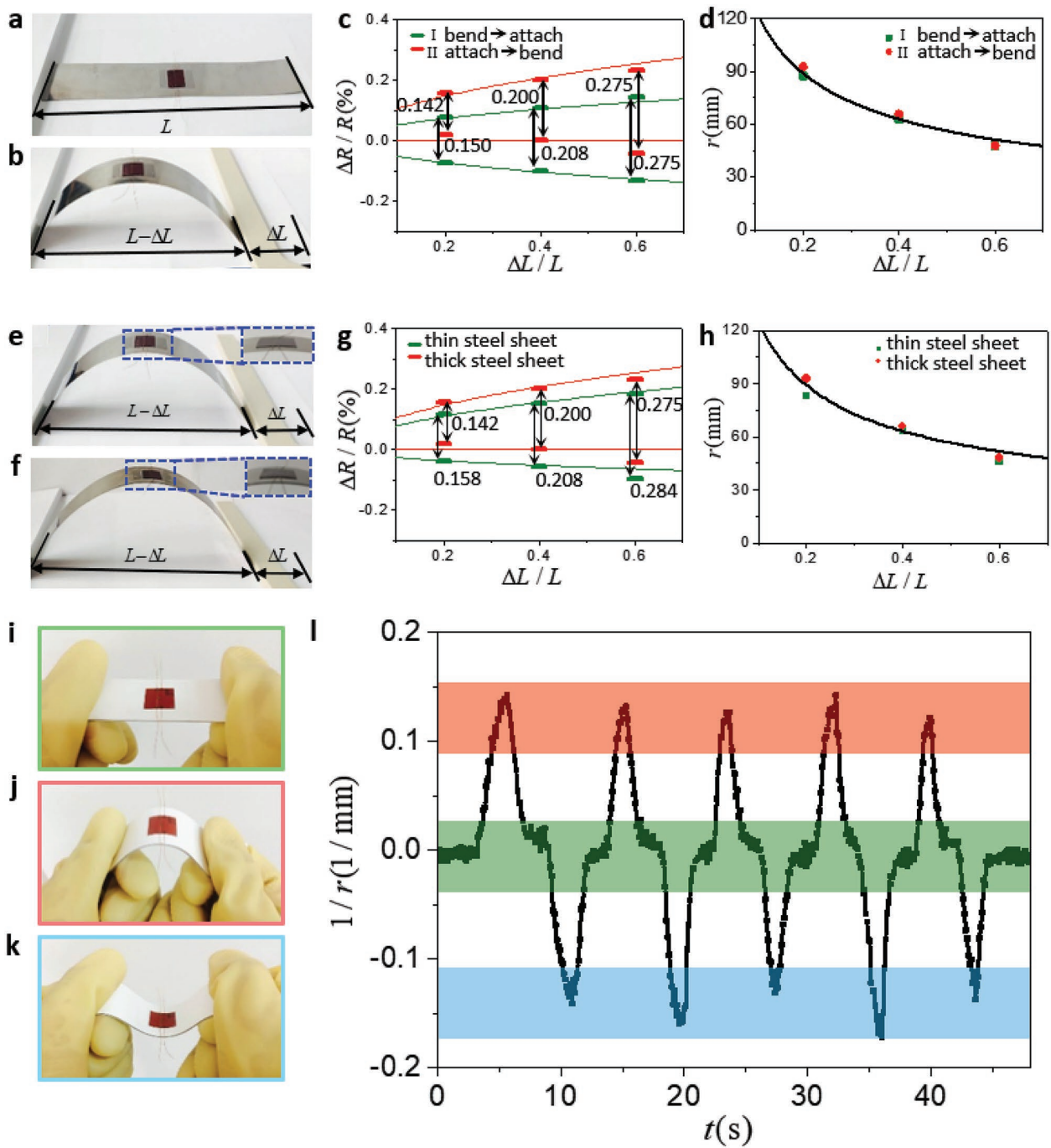

Figure 4. a) Attachment of the sensor to a steel sheet. b) Bending of the sheet by in-plane compression. c) Resistance changes for two ways of attaching. d) Comparison between the measured radii of curvature and those theoretical for two ways of attaching. Attachment of the sensor to e) a thin sheet, or f) a thick sheet. g) Resistance changes for two sheets with different thicknesses. h) Comparison between the measured radii of curvature and those theoretical for two sheets with different thicknesses. A further test for an object under periodic bending from i) the undeformed flat state to j) convex state, and to k) concave state (Movie S1, Supporting Information). I) Periodic time-varying curvature measurement.

recorded $\Delta R_{\text {top }} / R$ and $\Delta R_{\text {bottom }} / R$ for case I (green hyphens) have the same absolute values but opposite signs, while those for case II (red hyphens) shift upward by a certain value, which reflects the effect of surface strain $\varepsilon_{\text {surface }}$ These exprimental tests (hyphens) agree very well with the preditions from Equations (7) and (8) (lines). Although the measured resistance changes are different in the two cases, the same curvatures can be obtained, which can be theoretically shown by the substitution of Equations (7) and (8) into Equation (3). Experimental agreement is also observed in Figure 4c, e.g., 0.150 for case I versus 0.142 for case II, 0.208 versus 0.200 , and 0.275 versus 0.275 . The tested curvatures are accordingly compared with the theoretical ones, as plotted in Figure $4 d$, where satisfactory agreement is observed between the measured radii of curvature (dots) and those by Equation (5) (line). This confirms that the measurement of the present curvature sensor is independent of the ways of attaching, i.e., the surface strain.
Another group of experimental tests are carried out to further confirm that the measured curvature does not depend on the surface strain $\varepsilon_{\text {surface }}$ and the interfacial adhesion. Equation (6) shows that $\varepsilon_{\text {surface }}$ depends on the thickness of the steel sheet $t_{\text {sheet }}$. Therefore, the curvature sensors are used on two sheets with different thicknesses: a thin sheet with thickness of $0.05 \mathrm{~mm}$ (Figure 4e) and a thick sheet with thickness of $0.1 \mathrm{~mm}$ (Figure $4 \mathrm{f}$ ). Both are attached at the center before the sheet is bent (case II). Similar agreements on the difference of resistance changes (Figure $4 \mathrm{~g}$ ) and radii of curvature (Figure $4 \mathrm{~h}$ ) to those shown in Figure $4 \mathrm{c}, \mathrm{d}$ are found. It is confirmed that the measurement by the present sensor is scarcely influenced by the surface strain of the object itself.

In some situations, periodic bending is unavoidable, where both positive and negative curvatures need to be monitored. Typical examples include bending of a thumb, wiggle of a wrist, motion of a robot arm, etc. ${ }^{[31,32]}$ The capability of real-time 
monitoring is also absolutely essential in these cases. In this context, a further test is carried out for an object under periodic bending, with a period changing from the undeformed flat state (Figure 4i) to convex state (Figure 4j), and to concave state (Figure 4k), then back to the flat state (Movie S1, Supporting Information) within a couple of seconds, which coincides with the speeds of usual body movements. The measurement shown in Figure 41 gives the periodic time-varying curvature, encompassing both positive and negative characteristics, demonstrating the ability of the present sensor to accomplish both plus/minus and real-time curvature monitoring with good sensitivity.

\subsection{Representative Applications to Dynamic Curvature Monitoring}

Dynamic curvature measurement is of great significance in monitoring diverse body activities and measuring biomechanical signals toward healthcare applications. ${ }^{[33,34]}$ In view of the excellent performance elaborated in the previous section, we implement several representative dynamic curvature monitoring using our curvature sensors.

Cardiac physiological mapping is critically important in both basic and clinical cardiology. Figure 5a shows a recently developed representative $3 \mathrm{D}$ multifunctional integumentary
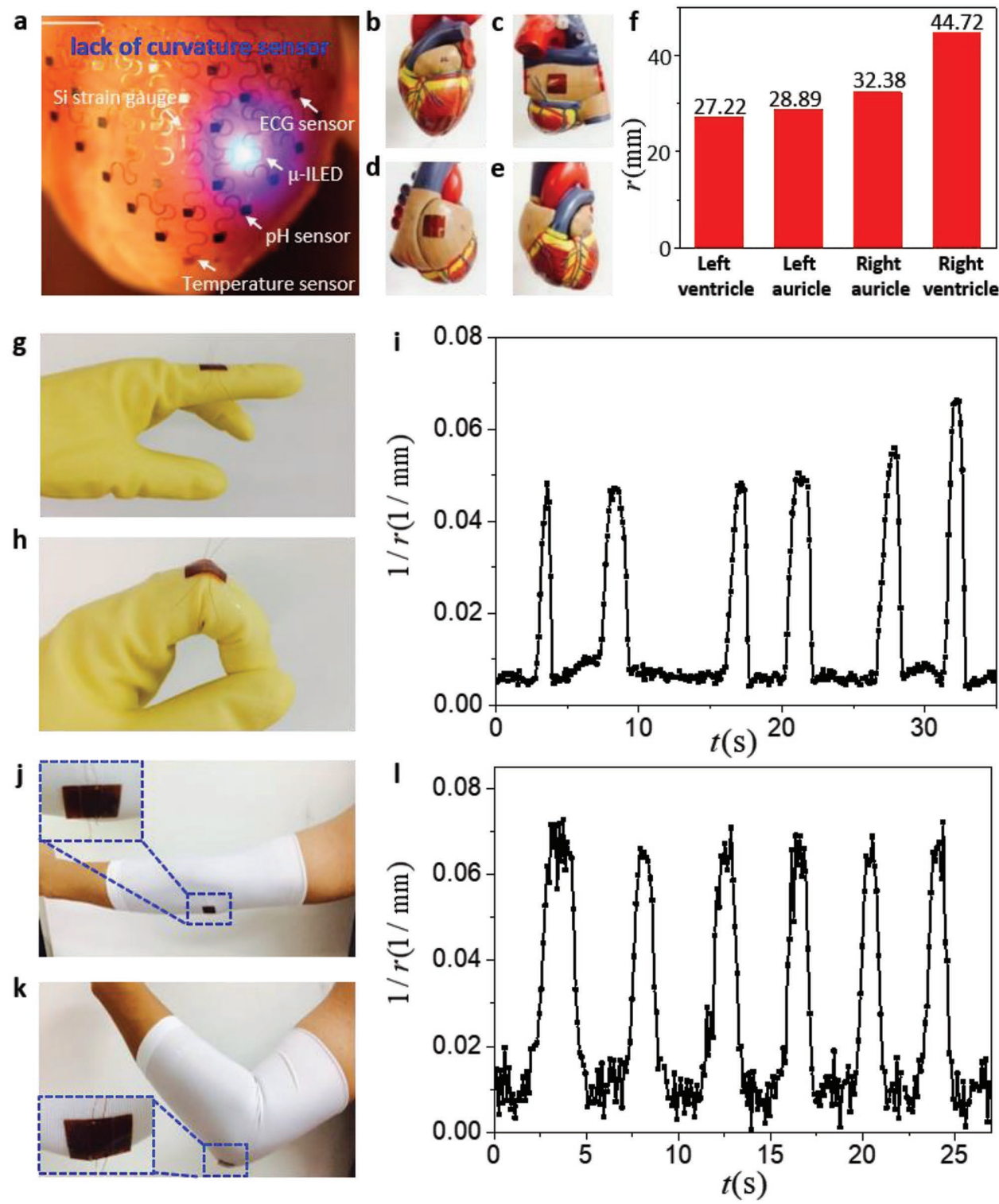

Figure 5. a) A recently developed representative 3D multifunctional integumentary membrane integrated on a Langendorff-perfused rabbit heart. Reproduced with permission from ref. [35]. b-e) Attachment of the sensors to both the left and right ventricles and atriums of a cardiac model. $f$ ) Histogram summarizing the radii of curvature of the four parts as shown in (b)-(e). g,h) Attachment of the sensor to a joint of a periodically bent index finger (Movie S2, Supporting Information). i) Curvature change with time for the joint of an index finger. j,k) Attachment of the sensor to a periodically bent elbow joint. l) Curvature change with time for the elbow joint. 
membrane integrated on a Langendorff-perfused rabbit heart, ${ }^{[35]}$ where various biosensors were used for measurements of cardiac activities, including sensors for $\mathrm{pH}$, temperature, mechanical strain, etc. It is noted that only the strain sensor was integrated for deformation sensing. However, because of the inevitable slippage of the device on the epicardial surface, accurate measurement of cardiac strain (i.e., tension/compression deformation) is unattainable, and the bending deformation cannot even be captured. By comparison, using the present curvature sensor can well address the above issues in view of the insensitivity to slippage. As a schematic demonstration, Figure 5b-e shows the curvature sensor that is attached to either the left or right ventricles and atriums of a cardiac model via the optically clear adhesive. Figure $5 \mathrm{f}$ plots the histogram that specifically summarizes the radii of curvature of the four parts, indicating that the quantitative capture of bending deformation is feasible by using the present sensor.

Monitoring joint activities plays an important role in human health monitoring, with potential applications to postoperative rehabilitation, gesture recognition, motion detection, etc. ${ }^{[36-38]}$ It may even provide more exciting opportunities in the field of artificial intelligence, e.g., by offering accurate sensing to help realize the simulated perception. ${ }^{[8,39-41]}$ The next application is thus on a joint of an index finger (Figure $5 \mathrm{~g}$ ). The curvature change with time is acquired during the periodic bending of the finger (Figure 5h), and is exported to Figure 5i (Movie S2, Supporting Information). Another similar application is on measuring the curvature of a periodically bent elbow joint. Figure $5 \mathrm{j}, \mathrm{k}$ corresponds to the states with zero and maximum curvatures, respectively, and the curvature-time curve is plotted in Figure 5l. With the inherent adhesion-free feature, the feasibility of integrating the present curvature sensor on wearable electronic devices is well illustrated by the above applications.

Successful capture of the real-time curvature information confirms the validity of the developed sensor for dynamic monitoring. It is worth noting that there may exists a difficulty in measuring the bending angles of some big joints with irregular surfaces such as the knee joint. ${ }^{[42-44]}$ The sensor itself must be long enough in such an application so that the full joint is covered. Since the surface curvature is not constant everywhere, the sensor will accordingly have variable curvature except at both ends where straight segments are maintained. The key problem arises: is the measurement using the present sensor still valid? Figure S2 (Supporting Information) shows the schematic illustration of a theoretical model of the sensor incorporating the effect of variable curvature distribution for measuring the bending angles of the joints. The sensor, having the apparent lengthl(the same size as that of the sensitive grids), is deformed into arbitrary shape due to the irregular surfaces of the joints such that the curvature changes with the curvilinear coordinate $s$ running from $A$ to $B$. For infinitesimally small $\mathrm{d} s$ at any point on $A B$, we have

$\mathrm{d}\left(\Delta R_{\text {top }}\right)=K_{\mathrm{s}} \varepsilon_{\text {top }} \mathrm{d} R$

$\mathrm{d}\left(\Delta R_{\text {bottom }}\right)=K_{\mathrm{s}} \varepsilon_{\text {bottom }} \mathrm{d} R$

at the top and bottom sides of the sensor, respectively. Here, each symbol is in accordance with that in the previous descriptions. The resistance change caused by bending is obtained by integration of Equation (9). Applying the relationships $\mathrm{d} R / d s=R / l, \mathrm{~d} \theta / \mathrm{d} s=1 / r$, and Equation (4), we have

$$
\left.\begin{array}{c}
\Delta R_{\mathrm{top}} \\
\Delta R_{\mathrm{bottom}}
\end{array}\right\}= \pm \frac{t K_{\mathrm{S}} R}{2 l}\left(\theta_{\mathrm{B}}-\theta_{\mathrm{A}}\right)
$$

From Equation (10), we have $\theta_{\mathrm{B}}-\theta_{\mathrm{A}}=l\left(\Delta R_{\text {top }}-\Delta R_{\text {bottom }}\right) /$ $\left(t K_{\mathrm{S}} R\right)$. Considering Equation (3), we write

$\theta_{\mathrm{B}}-\theta_{\mathrm{A}}=l \bar{\kappa}$

Here $\bar{\kappa}=\left(\Delta R_{\text {top }}-\Delta R_{\text {bottom }} /\right)\left(t K_{\mathrm{S}} R\right)$ represents the mean curvature, which is obtained by the measured resistance changes. Equation (11) suggests that the bending angle $\theta_{\mathrm{B}}-\theta_{\mathrm{A}}$ depends only on the apparent length of the sensor and mean curvature that is obtained by Equation (3). Therefore, the present sensor is still valid for measuring the bending angles of the joints with irregular surfaces such as the knee joint.

To demonstrate a device-level application of the adhesionfree curvature sensor, we fabricate a smart glove for gesture recognition in terms of curvature measurements (Figure 6a). Such a device is very helpful for facilitating fine-motion control in robotics and virtual reality; it might be used, e.g., as a master-hand to control a remote slave robot for telesurgery or to increase demining safety and speed. ${ }^{[4,27,28,45,46]}$ Here, the sensors are mounted on a soft stretchable glove via the optically clear adhesive, with the position of each sensor aligning with each knuckle of the fingers. The configurations of the sensors are given on the left and right of Figure 6a (see also Figure S3 and Table S2, Supporting Information for details). The serpentine stretchable structure (right middle image of Figure 6a) interconnects the sensors, and their assembly connects with a data processing module (right bottom image of Figure 6a) on the wrist top, which encompasses a tiny electronic display, a bluetooth module, five buzzers as well as LED indicators associated with the five fingers, and some necessary data processing chips. The measured resistance is recorded on the display, with $0.2 \Omega$ accuracy. The threshold can be set such that the buzzing sound is made once the difference of resistance changes exceeds the specified value, i.e., excessive curvature is reached. Figure $6 \mathrm{~b}$ gathers and plots the real-time curvatures for finger movements of a left hand, with the thumb, index, middle, both index and middle, both ring and little, and index-middle-ring group bent in sequence within $52 \mathrm{~s}$. The present curvature sensor-integrated smart glove can individually capture each finger's movement at different stages, demonstrating accurate response and favorable effectiveness of the glove. The sign language recognition can also be realized by detecting the curvature changes for different gestural expressions, e.g., indicating the letters "A," “C," “D," "E," "V," "Y," and the words "thank you," "20," and "good night," as demonstrated in Figure 6c. The device works well even under hundreds of bending cycles in our experiment, thus showing good robustness of the adhesion of the sensors to the glove. For a final commercial product, better adhesive and glove materials as well as encapsulation could be explored, which would further improve the robustness. The characteristic of clear differentiation among the gestures by the developed glove provides a new way of automatic 
a

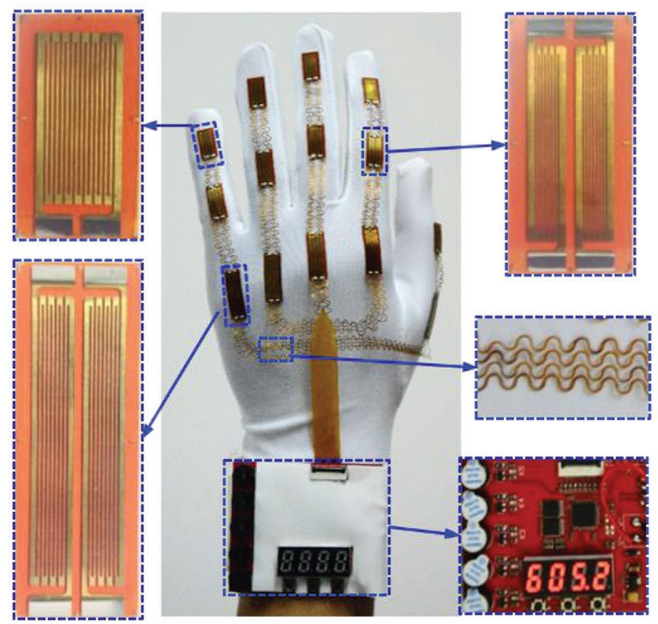

b
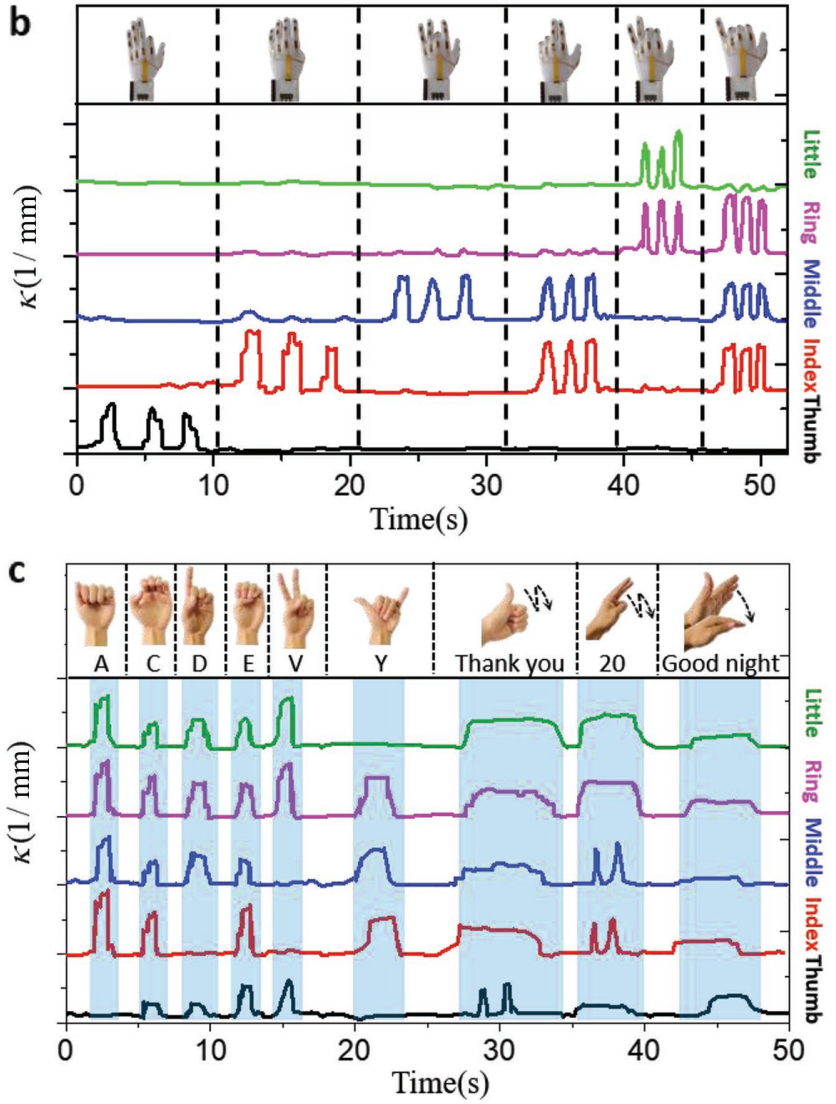

Figure 6. A smart glove for gesture recognition in terms of curvature measurements. a) Configuration of the sensors on the glove. b) Real-time curvatures for finger movements of a left hand, with the thumb, index, middle, both index and middle, both ring and little, and index-middlering group bent in sequence. c) Sign language recognition, with typical gestural expressions demonstrated.

understanding in the deaf community. Such a smart glove is also very useful for monitoring the finger motions of the patients in a minimally conscious or vegetative state, which is expected to play a remarkable role in wakening the patients.

A further application of the present curvature sensor is on the sitting posture monitoring and correction system, which is assembled on tight clothing to fulfill conformal movements with the human body. Such a device is of great significance for protecting people against diseases by incorrect sitting, e.g., cervical and lumbar spondylopathy, in a more comfortable way than conventional clumsy mechanical devices. ${ }^{[47]}$ Detailed configuration and dimensions appear in Figure S4 and Table S3 (Supporting Information), respectively. In Figure 7a (right image), an adult human subject, sitting upright in a chair from the start, bends down, then goes back to the upright state to finish a period of the bending movement. The curvatures at different locations of the back of the subject during bending are recorded by four curvature sensors that are attached to the positions corresponding to cervical (black), top and bottom thoracic (red and green), and lumbar vertebrae (blue), respectively (middle image of Figure 7a). The measured results are reflected by the curvature-time relationship as plotted in the left image of Figure 7a. Another similar measurement is carried out for periodic motions between upright upper body and drooping of the head of the subject (Figure 7b). An illustration of the measurements can be viewed in Movie S3 (Supporting Information). The difference between the two movements can be accurately captured by inspecting obvious changes in the curvature results; this shows that the present measurements are very sensitive, which is very useful for accurate correction of sitting postures. It is noted in the experiments that the thickness of the interlayer should be neither too thick, which may yield large strain in the gauges toward fracture, nor too thin, which may yield insensitivity of the sensor.

\subsection{Rosette Curvature Sensor}

The aforementioned sensor is only applicable to unidirectional curvature sensing, but the curvatures measured in different directions may differ for a complex surface, thus necessitating the introduction of a variant sensor that can sense multidirectional curvatures. Inspired by the design of rosette strain gauge, ${ }^{[48,49]}$ we propose a new rosette curvature sensor. For convenience, we focus on the design with two orthogonal sensitive grids at the top and two at the bottom of the interlayer (Figure 8a). The fabrication process is very similar to that of the unidirectional sensor as outlined in Figure 2d. Figure 8b shows such a sensor that is attached to a sphere. The solder tabs are designed to have relatively large cross-sectional area, by increasing the width (Figure 8c), and thus to have low electrical resistance such that they are insensitive to strain, which avoids the impact on the strain-sensitive grids. Detailed configuration and dimensions appear in Figure S5 and Table S4 (Supporting Information), respectively. Calibration of the rosette curvature sensor is implemented as proceeded in Figure 3c, except that the bending is conducted in both orthogonal directions. As shown in Figure 8d, bending in the vertical direction gives a set of nonzero results for the vertical sensitive grids (measurement 1), yielding the GF of $\approx 1.578$ that is the same as that obtained from Figure $3 \mathrm{c}$, while zero resistance change is found for the horizontal sensitive grids (measurement 2), indicating that the measurements in two directions are independent. Similar results are found for bending in the horizontal direction (Figure 8e), giving the same GF. The measurements on 

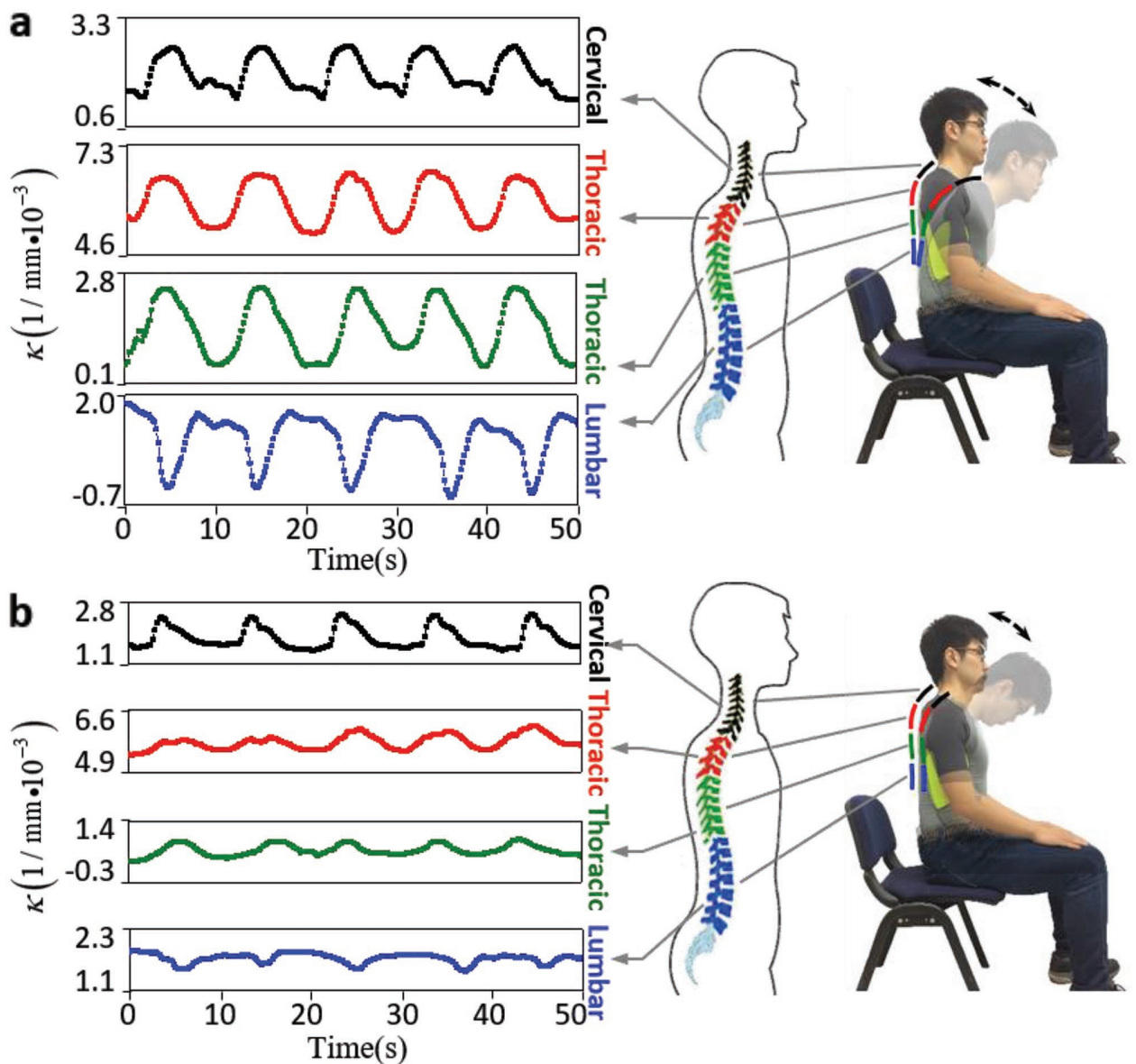

Figure 7. Application of the curvature sensor to sitting posture monitoring and correction. a) Measurement of periodic motions between upright sitting and bending down of the upper body of an adult human subject. b) Measurement of periodic motions between upright sitting and drooping of the head of the subject. See Movie S3 (Supporting Information) for a larger field of view of the measurements.

two spheres with different radii of curvature indicate very small errors of both orthogonal gauges (Figure $8 \mathrm{f}$ ). The periodic concave (Figure $8 \mathrm{~g}$ ) and convex (Figure $8 \mathrm{~h}$ ) diagonal bending of the rosette curvature sensor attached to an object is carried out. The real-time monitoring in Figure $8 \mathrm{i}$ confirms that both orthogonal gauges yield very close curvatures in the $45^{\circ}$ direction.

\section{Conclusion}

In this study, we introduce a novel adhesion-free thin-filmlike curvature sensor that can monitor bending activities when integrated with flexible and stretchable platforms. The adhesion-free tests and sample curvature measurements confirm the prominent feature that the measurement is independent of the strain of the target surface and the interfacial slippage, i.e., perfect adhesion between the sensor and the surface is unnecessary. A periodic bending test demonstrates the ability to accomplish both plus/minus and real-time curvature monitoring with good sensitivity. With the well-established characters, the present sensor is extensively applied for quantitative monitoring of bending deformation, including measurements of a cardiac model and various joint activities. The devicelevel demonstrations reveal the comprehensive abilities of the sensor, including a smart glove for gesture recognition and a sitting posture monitoring and correction system. Multidirectional curvature sensing is also fulfilled by a new design of rosette strain gauge for monitoring complex surfaces. The comprehensive design, fabrication, mechanism, structural analysis, performance characterization, and device-level demonstrations of the sensor as reported in this study highlight its advantages of adhesion-free, simple mechanical principle, low cost, and satisfactory monitoring results, thus showing exceptional superiority for applications to various flexible and wearable electronic devices.

\section{Experimental Section}

Fabrication Process of Adhesion-Free Flexible Curvature Sensor: The fabrication process is shown in Figure 2d. The constantan foil for the sensitive grids is composed of $59 \%$ copper, $40 \%$ nickel, and $1 \%$ manganese, which was cut into a size larger than that of the mask. The acetone solution was used to remove the contaminations on the surface of the foil. As the adhesion promoter, novolac epoxy was coated on the foil surface, followed by placing it over a hot plate while stabilizing the temperature at $150^{\circ} \mathrm{C}$ for $2 \mathrm{~h}$ to accelerate solidification. Conventional photolithography was adopted to produce the sensitive grids, i.e., spin coating of photoresist, exposure, developing, etching 

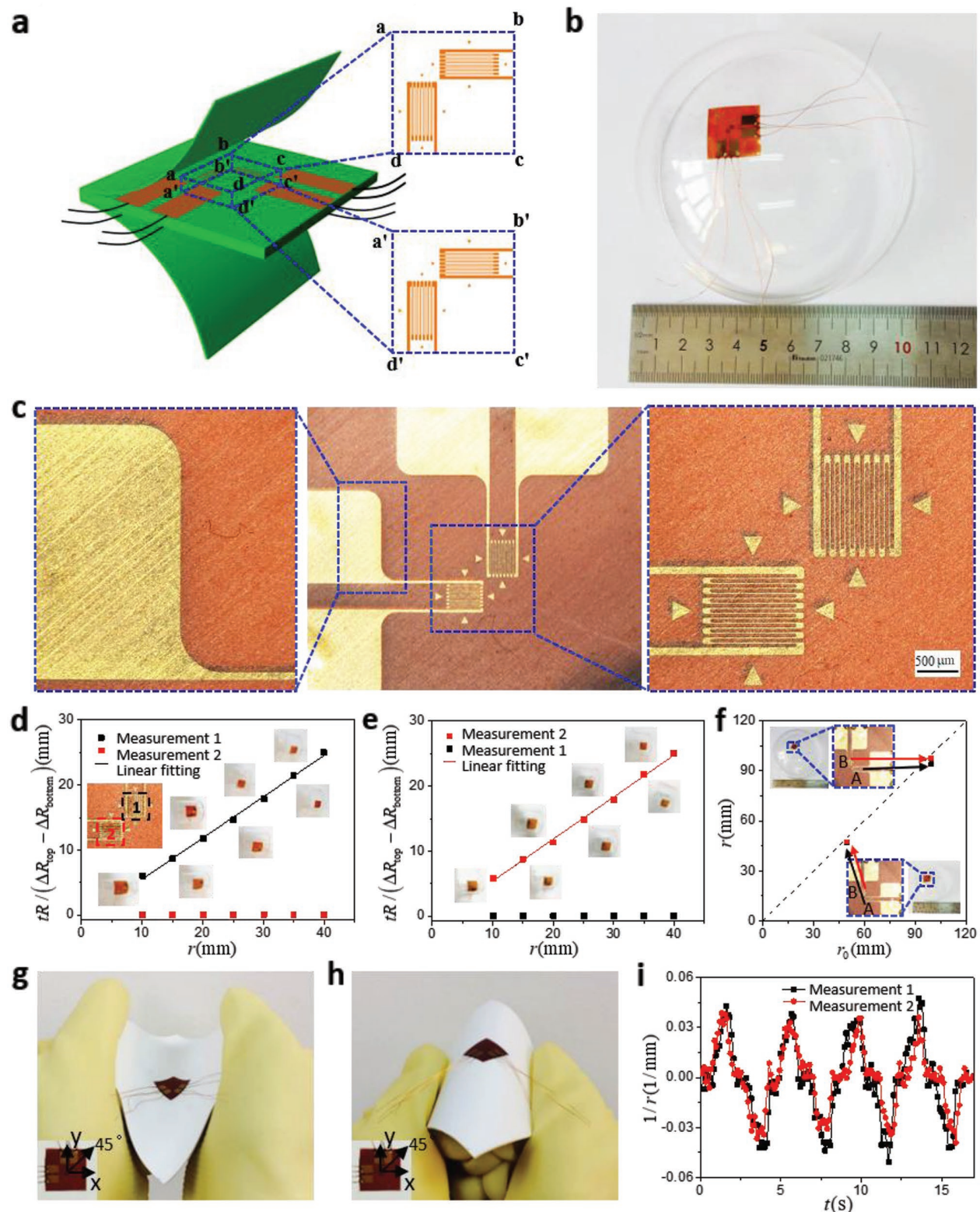

Figure 8. A new rosette curvature sensor. a) Exploded view of the rosette sensor with orthogonal sensitive grids. b) Demonstration of the rosette curvature sensor that is attached to a sphere. c) Top-view optical image of the sensitive grids. d,e) Differences of resistance changes with sphere radius for bending in both orthogonal directions. f) Measurements from both orthogonal gauges on two spheres with different radii of curvature. g,h) Periodic concave and convex diagonal bending of the rosette curvature sensor that is attached to an object. i) Real-time monitoring of curvatures in the $45^{\circ}$ direction.

of exposed foil (by ferric chloride solution), and removal of remaining photoresist (by trichloroethylene). The configuration of the generated sensitive grids was inspected by a high-powered microscope, then the diluted corrosive ferric chloride solution in combination with mechanical polish was used to adjust the resistance to $120 \Omega$. A durable protecting layer was then stuck to the sensitive grids. In the next step, the external leads were weld to the end of the grids. After cutting into the size as desired and conducting the configuration and resistance examinations, two sensitive grids were integrated with perfect reverse alignment on both sides of the interlayer by thermocompression bonding. Two encapsulation layers were laminated on the top and bottom of the interlayer with the sensitive grids to finish the fabrication. Informed signed consent was obtained from the subject involved in the experiments.
Finite Element Analysis: The FEA was conducted using the ABAQUS software package for bending of a part of the sensor (Figure $3 \mathrm{~b}$ ). The Young's moduli and Poisson's ratios for the materials used were $2.5 \mathrm{GPa}$ and 0.35 for $\mathrm{PI}$ (encapsulated layers), $4 \mathrm{GPa}$ and 0.35 for phenolic resin (interlayer), and $163 \mathrm{GPa}$ and 0.33 for constantan (sensitive grids). ${ }^{[50,51]}$ The entire part was tied to two rigid bodies at both ends, and a rotation angle was imposed on the rigid bodies to realize the pure bending of the model. The 8-node linear brick element was adopted for the laminate.

\section{Supporting Information}

Supporting Information is available from the Wiley Online Library or from the author. 


\section{Acknowledgements}

The authors gratefully acknowledge the support from the National Natural Science Foundation of China (grant nos. 11772331, 11572323, 11302038,11622214 , and 11472027). Y.S. acknowledges the support from Chinese Academy of Sciences via the "Hundred Talent Program," Strategic Priority Research Program of the Chinese Academy of Sciences (grant no. XDB22040501). Y.C. acknowledges the Program for New Century Excellent Talents in University (grant no. NCET-13-0021). R.L. acknowledges the support from the Young Elite Scientists Sponsorship Program by CAST (grant no. 2015QNRC001), Opening Fund of State Key Laboratory of Nonlinear Mechanics, Chinese Academy of Sciences, and Fundamental Research Funds for the Central Universities of China (grant no. DUT18GF101)

\section{Conflict of Interest}

The authors declare no conflict of interest.

\section{Keywords}

adhesion-free, curvature sensor, flexible electronics, stretchable electronics

Received: August 2, 2018 Revised: September 24, 2018 Published online:

[1] T. Someya, T. Sekitani, S. Iba, Y. Kato, H. Kawaguchi, T. Sakurai, Proc. Natl. Acad. Sci. USA 2004, 101, 9966.

[2] T. Someya, Y. Kato, T. Sekitani, S. Iba, Y. Noguchi, Y. Murase, H. Kawaguchi, T. Sakurai, Proc. Natl. Acad. Sci. USA 2005, 102, 12321.

[3] M. C. Mcalpine, H. Ahmad, D. Wang, J. R. Heath, Nat. Mater. 2007, 6, 379 .

[4] T. Yamada, Y. Hayamizu, Y. Yamamoto, Y. Yomogida, A. Izadi-najafabadi, D. N. Futaba, K. Hata, Nat. Nanotechnol. 2011, 6, 296.

[5] C. Wang, K. Xia, M. Zhang, M. Jian, Y. Zhang, ACS Appl. Mater. Interfaces 2017, 9, 39484.

[6] C. Wang, K. Xia, M. Jian, H. Wang, M. Zhang, Y. Zhang, J. Mater. Chem. C 2017, 5, 7604

[7] M. Zhang, C. Wang, H. Wang, M. Jian, X. Hao, Y. Zhang, Adv. Funct. Mater. 2017, 27, 1604795.

[8] J. Kim, M. Lee, H. J. Shim, R. Ghaffari, H. R. Cho, D. Son, Y. H. Jung, M. Soh, C. Choi, S. Jung, K. Chu, D. Jeon, S. T. Lee, J. H. Kim, S. H. Choi, T. Hyeon, D. H. Kim, Nat. Commun. 2014, 5, 5747.

[9] B. U. Hwang, J. H. Lee, T. Q. Trung, E. Roh, D. I. Kim, S. W. Kim, N. E. Lee, ACS Nano 2015, 9, 8801.

[10] M. Corinne, C. Frank, T. Gerhard, Sensors 2008, 8, 3719.

[11] N. Lu, C. Lu, S. Yang, J. Rogers, Adv. Funct. Mater. 2012, 22, 4044.

[12] S. Ryu, P. Lee, J. B. Chou, R. Xu, R. Zhao, A. J. Hart, S. G. Kim, ACS Nano 2015, 9, 5929.

[13] Z. Liu, D. Qi, P. Guo, Y. Liu, B. Zhu, H. Yang, Y. Liu, B. Li, C. Zhang, J. Yu, B. Liedberg, X. Chen, Adv. Mater. 2015, 27, 6230.

[14] Y. Cheng, R. Wang, J. Sun, L. Gao, Adv. Mater. 2015, 27, 7365.

[15] C. Yan, J. Wang, W. Kang, M. Cui, X. Wang, C. Y. Foo, K. J. Chee, P. S. Lee, Adv. Mater. 2014, 26, 2022.

[16] C. Wang, X. Li, E. Gao, M. Jian, K. Xia, Q. Wang, Z. Xu, T. Ren, Y. Zhang, Adv. Mater. 2016, 28, 6640 .

[17] M. K. Choi, O. K. Park, C. Choi, S. Qiao, R. Chaffari, J. Kim, D. J. Lee, M. Kim, W. Hyun, S. J. Kim, H. J. Hwang, S. H. Kwon, T. Hyeon, N. Lu, D. H. Kim, Adv. Healthcare Mater. 2016, 5, 80.
[18] S. Choi, S. I. Han, D. Jung, H. J. Hwang, C. Lim, S Bae, O. K. Park, C. M. Tschabrunn, M. Lee, S. Y. Bae, J. W. Yu, J. H. Ryu, S. W. Lee, K. Park, P. M. Kang, W. B. Lee, R. Nezafat, T. Hyeon, D. H. Kim, Nat. Nanotechnol. 2018, https://doi.org/10.1038/s41565-018-0226-8.

[19] H. Jin, S. Jung, J. Kim, S. Heo, J. Lim, W. Park, H. Y. Chu, F. Bien, K. Park, Sci. Rep. 2017, 7, 10854.

[20] T. Lee, W. Lee, S. W. Kim, J. J. Kim, B. S. Kim, Adv. Funct. Mater 2016, 26, 6206.

[21] R. Xu, Y. Lu, C. Jiang, J. Chen, P. Mao, G. Gao, L. Zhang, S. Wu, ACS Appl. Mater. Interfaces 2014, 6, 13455.

[22] H. Lee, A. J. Rosakis, L. B. Freund, J. Appl. Phys. 2001, 89, 6116.

[23] Y. Gong, T. Zhao, Y. J. Rao, Y. Wu, IEEE Photonics Technol. Lett. 2011, 23, 679.

[24] D. Z. Stupar, J. S. Bajic, L. M. Manojlovic, M. P. Slankamenac, A. V. Joza, M. B. Zivanov, IEEE Sens. J. 2012, 12, 3424.

[25] S. P. Timoshenko, J. M. Gere, Theory of Elastic Stability, McGraw-Hill, Inc., New York, NY 1961.

[26] M. Hempel, D. Nezich, J. Kong, M. Hofmann, Nano Lett. 2012, 12, 5714.

[27] M. Amjadi, A. Pichitpajongkit, S. Lee, S. Ryu, I. Park, ACS Nano 2014, 8, 5154.

[28] J. Lee, H. Kwon, J. Seo, S. Shin, J. H. Koo, C. Pang, S. Son, J. H. Kim, Y. H. Jang, D. E. Kim, T. Lee, Adv. Mater. 2015, 27, 2433.

[29] Y. H. Lee, J. S. Kim, J. Noh, I. Lee, H. J. Kim, S. Choi, J. Seo, S. Jeon, T. S. Kim, J. Y. Lee, J. W. Choi, Nano Lett. 2013, 13, 5753.

[30] Y. Su, J. Wu, Z. Fan, K. C. Hwang, J. Song, Y. Huang, J. A. Rogers, J. Mech. Phys. Solids 2012, 60, 487.

[31] S. Lim, D. Son, J. Kim, Y. B. Lee, J. K. Song, S. Choi, D. J. Lee, J. H. Kim, M. Lee, T. Hyeon, D. H. Kim, Adv. Funct. Mater. 2015, 25, 375.

[32] A. K. Bansal, S. Hou, O. Kulyk, E. M. Bowman, I. D. W. Samuel, Adv. Mater. 2015, 27, 7638.

[33] A. V. Alaferdov, R. Savu, T. A. Rackauskas, S. Rackauskas, M. A. Canesqui, D. S. de Lara, G. O. Setti, E. Joanni, G. M. de Trindade, U. B. Lima, A. S. de Souza, S. A. Moshkalev, Nanotechnology 2016, 27, 375501.

[34] J. M. Williams, I. Haq, R. Y. Lee, Med. Eng. Phys. 2010, 32, 1043.

[35] L. Xu, S. R. Gutbrod, A. P. Bonifas, Y. Su, M. S. Sulkin, N. Lu, H. J. Chung, K. I. Jang, Z. Liu, M. Ying, C. Lu, R. C. Webb, J. S. Kim, J. I. Laughner, H. Cheng, Y. Liu, A. Ameen, J. W. Jeong, G. T. Kim, Y. Huang, I. R. Efimov, J. A. Rogers, Nat. Commun. 2014, 5, 3329.

[36] Y. C. Lai, J. Deng, S. L. Zhang, S. Niu, H. Guo, Z. L. Wang, Adv. Funct. Mater. 2017, 27, 1604462.

[37] B. An, Y. Ma, W. Li, M. Su, F. Li, Y. Song, Chem. Commun. 2016, 52, 10948.

[38] M. D. Bartlett, E. J. Markvicka, C. Majidi, Adv. Funct. Mater. 2016, 26, 8496.

[39] D. Floreano, R. Pericet-Camara, S. Viollet, F. Ruffier, A. Brückner, R. Leitel, W. Buss, M. Menouni, F. Expert, R. Juston, M. K. Dobrzynskia, G. L'Eplatteniera, F. Recktenwalde, H. A. Mallote, N. Franceschinib, Proc. Natl. Acad. Sci. USA 2013, $110,9267$.

[40] X. Wang, L. Dong, H. Zhang, R. Yu, C. Pan, Z. L. Wang, Adv. Sci. 2015, 2, 1500169.

[41] H. He, Y. Fu, W. Zang, Q. Wang, L. Xing, Y. Zhang, X. Xue, Nano Energy 2017, 31, 37.

[42] S. I. Lee, J. F. Daneault, L. Weydert, P. Bonato, in IEEE 13th Int. Conf. Wearable Implantable Body Sensor Networks, San Francisco, CA 2016, p. 377.

[43] M. Totaro, T. Poliero, A. Mondini, C. Lucarotti, G. Cairoli, J. Ortiz, L. Beccai, Sensors 2017, 17, 2314.

[44] G. Cai, J. Wang, K. Qian, J. Chen, S. Li, P. S. Lee, Adv. Sci. 2017, 4, 1600190

[45] S. Lee, S. Shin, S. Lee, J. Seo, J. Lee, S. Son, H. J. Cho, H. Algadi, S. Al-Sayari, D. E. Kim, T. Lee, Adv. Funct. Mater. 2015, 25, 3114. 
[46] A. P. Gerratt, H. O. Michaud, S. P. Lacour, Adv. Funct. Mater. 2015, 25, 2287.

[47] M. H. Pope, K. L. G. And, M. L. Magnusson, Annu. Rev. Biomed. Eng. 2002, 4, 49.

[48] S. H. Bae, Y. Lee, B. K. Sharma, H. J. Lee, J. H. Kim, J. H. Ahn, Carbon 2013, 51, 236.
[49] J. H. Kong, N. S. Jang, S. H. Kim, J. M. Kim, Carbon 2014, 77, 199.

[50] S. Xu, Y. Zhang, J. Cho, J. Lee, X. Huang, L. Jia, J. A. Fan, Y. Su, J. Su, H. Zhang, H. Cheng, B. Lu, C. Yu, C. Chuang, T. Kim, T. Song, K. Shigeta, S. Kang, C. Dagdeviren, I. Petrov, P. V. Braun, Y. Huang, U. Paik, J. A. Rogers, Nat. Commun. 2013, 4, 1543.

[51] Y. Su, S. Li, R. Li, C. Dagdeviren, Appl. Phys. Lett. 2015, 107, 3398. 